\title{
Identifying priorities in knowledge translation from the perspective of trainees: results from an online survey
}

Kristine Newman ${ }^{1 *}$, Dwayne Van Eerd ${ }^{2,3}$, Byron J. Powell ${ }^{4}$, Robin Urquhart ${ }^{5}$, Evelyn Cornelissen ${ }^{6}$, Vivian Chan $^{7}$ and Shalini Lal ${ }^{8}$

\begin{abstract}
Background: The need to identify priorities to help shape future directions for research and practice increases as the knowledge translation (KT) field advances. Since many KT trainees are developing their research programs, understanding their concerns and $\mathrm{KT}$ research and practice priorities is important to supporting the development and advancement of $K T$ as a field. Our purpose was to identify research and practice priorities in the KT field from the perspectives of $\mathrm{KT}$ researcher/practitioner trainees.

Findings: Survey response rate was $62 \%$ (44/71). Participants were mostly Canadian graduate students, post-doctoral fellows, residents, and learners from various disciplines; the majority was from Ontario (44\%) and Quebec (20 \%). Seven percent (5/71) were from other countries including USA, UK, and Switzerland. Seven main KT priority themes were identified: determining the effectiveness of KT strategies, technology use, increased key stakeholder involvement, context, theory, expand ways of inquiry, and sustainability.

Conclusions: Overall, the priorities identified by the trainees correspond with KT literature and with KT experts' views. The trainees appeared to push the boundaries of current $K T$ literature with respect to creative use of communication technologies research.
\end{abstract}

Keywords: Knowledge translation, Trainee, Research priorities

\section{Findings}

\section{Purpose and methods}

The knowledge translation (KT) field has advanced rapidly in the last decade. Given that KT trainees are developing programs of research, understanding their priorities and concerns for KT research and practice is useful to advancing the KT field. This report defines KT as the "iterative, timely, and effective process of integrating best evidence into the routine practice of patients, practitioners, health-care teams, and systems" [1]. Indeed, working with their mentors, they may identify novel ways of viewing KT challenges and new approaches to solving them. The purpose of this study was to identify priorities for KT research and practice from

\footnotetext{
* Correspondence: kristine.newman@ryerson.ca

${ }^{1}$ Daphne Cockwell School of Nursing, Faculty of Community Services,

Ryerson University, Toronto M5B 2K3, Canada

Full list of author information is available at the end of the article
}

the perspectives of KT researcher and practitioner trainees. Trainee perspectives can complement expert views expressed in the KT literature [2, 3].

A Web-based survey was used to identify KT trainees' priorities related to KT practice and research. The 22 items included demographics, geographic location, career stage, KT experience, KT research priorities, and KT topics unworthy of further research/exploration. A purposive sample of trainees affiliated with the KT Trainee Collaborative (KTTC) [4] and 2013 KT Canada Summer Institute [2] was e-mailed a survey link through FluidSurveys ${ }^{\mathrm{Tu}}$. Non-responders were sent three reminder emails 7, 14, and 21 days after the initial e-mail [5]. Responses to open-ended questions were analyzed using qualitative content analysis [6, 7]. The open-ended responses were reviewed and coded independently by two investigators [BJP, $\mathrm{VC}]$ who revised codes as general categories emerged. The codes were discussed and 
disagreements were reviewed in detail by coders until agreement was reached. The codes were then grouped thematically. The study team reviewed, discussed, and refined the themes to ensure they accurately represented the participants' responses.

The Hamilton Integrated Research Ethics Board approved the study procedures.

\section{Results}

We obtained a response rate of $62 \%(44 / 77)$, and the respondents completed $91 \%$ of the questions. The respondents self-identified as Masters or PhD students (60\%) or faculty (14\%), with the remainder (26\%) identifying as practitioners, clinician scientists, and fellows. Many respondents reported being involved in both $\mathrm{KT}$ research and practice (45\%) or solely KT research (41\%), with the remainder involved in KT practice or other (14\%).

The data were categorized into seven main themes related to KT priorities. Many of these priorities relate to one another, as depicted in Additional file 1.

\section{Determining the effectiveness of KT strategies}

The most frequently mentioned KT priority concerned developing generalizable knowledge about the effectiveness of KT strategies in various contexts. The respondents spoke to the need for more empirical testing of KT strategies ("I believe that there needs to be a greater number of good quality, theory-based KT interventional studies conducted to improve our understanding of uptake and spread of knowledge") [respondent D]. Many suggested testing whether tailored KT strategies are more effective than generic, one-size-fits-all strategies. The respondents emphasized the importance of adapting existing strategies from behavior change research ("many people do not benefit from this [the theoretical and empirical work related to behavior change] wisdom.") [respondent N].

\section{Use of technology}

The respondents frequently reported the need to capitalize on technologies such as the Web, mobile phone applications, health informatics, and social media in $\mathrm{KT}$ research and practice. One respondent wrote: "KT is about communication and the new technologies provide opportunities to test and understand the dilemma of the knowledge to practice gap differently" [respondent E]. The respondents highlighted the potential benefits and importance of sharing data through technology and online: "Online sharing is very easy, but the 'open source' mentality is far from the norm in ... health research. On a positive note, KT researchers are very open in general, since they value collaboration and interdisciplinarity" [respondent N].

\section{Increased involvement of key stakeholders}

The respondents noted the importance of more stakeholder involvement in various aspects of KT. This included developing partnerships with key stakeholders, such as commissioning bodies and a broad range of end users (e.g., policy makers, health-care providers, and patients) as well as across countries. One respondent stated, "it is not always clear either to practitioners that their particular local/ lay knowledge is always welcome in academic research" [respondent AA]. Similarly, as another stated, "we often forget our main partner, the patient...there is a world that we need to explore there" [respondent K]. Lastly, the respondents acknowledged that stakeholder participation is limited by academic culture, which does not reward academic researchers for participating in KT practice. As one respondent $[\mathrm{F}]$ stated:

Academia creates incentives for publication, which we know is not an adequate strategy for effective KT. Creating solid partnerships takes a lot of time and resources, but those efforts are not valued and recognized in academia.

\section{Importance of context}

The respondents prioritized assessing contextual elements in two ways. First, the respondents suggested that research is needed to clarify contextual constructs and develop methods for collecting, analyzing, and acting on data about contexts. For instance, the respondents prioritized pre-implementation assessments, implementation barriers, and feasibility of implementation. Second, many respondents suggested that specific contexts (or settings) require more $\mathrm{KT}$ research, such as dementia care, primary care, nutrition, chronic care, health-care organizations, rural settings, and low-resourced settings. For example, one respondent $[\mathrm{F}]$ challenged the $\mathrm{KT}$ research community to pay more attention to the needs of Indigenous peoples:

I believe the CIHR model for KT does not go far enough in recognizing and valuing different cultural (and other) factors that are necessary for effective KT with our Indigenous people-a true ethical problem in a time when health problems are dire for many Indigenous communities.

Finally, the respondents recommended that more attention be given to how evidence can be adapted to local contexts.

\section{Importance of theory}

Several respondents' priorities related to the use of theory in KT research and practice, stating that using theory is a prerequisite for quality KT research. They 
expressed their belief that using theories from diverse disciplines and perspectives is important and also suggested using "theoretical frameworks to identify the "key components' or drivers in knowledge exchange" [respondent AJ]. While some respondents suggested specific theories (e.g., complexity theory), others referred more generally to the importance of integrating theory and practice.

Sixteen of the 44 respondents responded that there needs to be less emphasis on developing new KT frameworks/models and more emphasis on testing, refining, and improving those that already exist.

\section{Expand our ways of inquiry}

Many respondents identified research priorities related to expanding our ways of inquiry. One respondent felt "we need to explore other modes of inquiry that are more finely attuned to the particular that shed light on specific relationships between actual people" [respondent X]. In addition, many suggested embracing a broader array of research methodologies and approaches underutilized in KT research, such as social network analysis, economic evaluation, mixed methods, and qualitative research. As one respondent $[\mathrm{AL}]$ noted,

$\mathrm{KT}$ is predominantly supported from a more traditional research perspective yet continuously, research indicates that the most critical component to the success or failure of a KT project or strategy is contextual. Qualitative research can more effectively get at context.

The respondents also prioritized development of valid and reliable outcome measures, including those for contextual elements (e.g., organizational readiness for change), complex interventions, service-system outcomes (e.g., timeliness), implementation outcomes (e.g., sustainability), and downstream effects of KT efforts on end users and health-care teams. They also expressed the importance of using evaluation frameworks and of routinely conducting evaluations of $\mathrm{KT}$ efforts.

Finally, the respondents prioritized improved descriptions of $\mathrm{KT}$ processes and research through development of reporting guidelines specific to KT. Reporting guidelines were suggested in response to a perceived problem in the literature: "limitations of syntheses are often related to intervention reporting" [respondent $\mathrm{M}$ ].

\section{Sustainability}

The respondents indicated that sustainability is a top priority at the design phase of any KT research or practice initiative. Many mentioned measuring sustainability of KT efforts as a priority. One respondent acknowledged, "We don't know if it is our efforts are sustainable, or even if they should be" [respondent K]. Other respondents noted the need for the development and testing of specific KT strategies to enhance sustainability.

\section{Conclusions}

Overall, KT trainees identified $\mathrm{KT}$ research and practice priorities that align closely with those noted by KT experts in the KT literature. These include understanding context and contextual factors [8,9], using theory in research and practice [10-13], evaluating effectiveness of KT strategies [3, 14-17], considering factors related to sustainability $[18,19]$, and employing new approaches to evaluation or ways of inquiry to better understand $\mathrm{KT}$ [3, 20-25]. Specifically, Eccles et al. [3] noted that identification, development, refinement, and testing of $\mathrm{KT}$ strategies have been prioritized by federal governments and there have been calls to utilize mixed methods and qualitative approaches to understand the nuances of contexts and processes related to $\mathrm{KT}$. We determined trainee priorities through an empirical process to complement the views of experienced KT experts. These priorities reinforce the need to move $\mathrm{KT}$ research and practice forward in a number of strategic areas. These priorities will certainly not be the last word. Attending to these areas creatively will undoubtedly lead to the identification of further priorities and, in the process, help to strengthen the science and practice of KT.

\section{Additional file}

Additional file 1: Identified KT Priorities. Categorized seven main

themes related to $\mathrm{KT}$ priorities

\section{Abbreviations}

KT: knowledge translation; KTSI: 2013 KT Canada Summer Institute; KTTC: Knowledge Translation Trainee Collaborative.

\section{Competing interests}

The authors declare that they have no competing interests.

\section{Authors' contributions}

All authors contributed substantially to the (1) conception and design of the study, (2) analysis and interpretation of the data, and (3) critical review and revision of the manuscript. They have given final approval of the version to be published and agreed to be accountable for all aspects of the work. KN and DVE also acquired funding and coordinated the study, and KN completed data collection.

\section{Acknowledgements}

We would like to acknowledge the respondents for their participation in the study. This research was partly funded by the Faculty of Community Services, Ryerson University by the Spring 2014 Publication Grant and in part by a CIHR Planning Grant \#119110. We thank McMaster University, Faculty of Health Sciences, School of Nursing for ethics assistance. We thank the Dean's Office, Faculty of Community Services, Ryerson University for funding professional editing of this article.

\section{Author details}

'Daphne Cockwell School of Nursing, Faculty of Community Services,

Ryerson University, Toronto M5B 2K3, Canada. ${ }^{2}$ Institute for Work and Health, Toronto, Canada. ${ }^{3}$ School of Public Health and Health Systems, University of Waterloo, Waterloo, Canada. ${ }^{4}$ Department of Psychiatry, Perelman School of 
Medicine, University of Pennsylvania, Pennsylvania, USA. ${ }^{5}$ Department of Surgery, Dalhousie University, Halifax, Canada. ${ }^{6}$ Department of Family Practice, Faculty of Medicine, University of BC, Vancouver, BC, Canada. ${ }^{7}$ Department of Medicine, Quality and Safety, Vancouver Coastal Health, Vancouver, Canada. ${ }^{8}$ School of Rehabilitation, Faculty of Medicine, University of Montreal, Montreal, Canada.

Received: 29 January 2015 Accepted: 18 June 2015

Published online: 21 June 2015

\section{References}

1. Davis D. Continuing education, guideline implementation, and the emerging transdisciplinary field of knowledge translation. J Contin Educ Health Prof. 2006;26:5-12.

2. Straus SE, Brouwers M, Johnson D, Lavis JN, Legare F, Majumdar SR, et al. Core competencies in the science and practice of knowledge translation: description of a Canadian strategic training initiative. Implement Sci. 2011;6:127.

3. Eccles MP, Armstrong D, Baker R, Cleary K, Davies H, Davies S, et al. An implementation research agenda. Implement Sci. 2009;4(18):1-7. doi:10.1186/1748-5908-4-18.

4. Urquhart R, Cornelissen E, Lal S, Colquhoun H, Klein G, Richmond S, et al. A community of practice for knowledge translation trainees: an innovative approach for learning and collaboration. J Contin Educ Health Prof. 2013;33(4):274-81.

5. Dillman DA, Smyth JD, Christian L. Internet, mail, and mixed-mode surveys: the tailored design method. Hoboken NJ: John Wiley \& Sons Inc; 2008.

6. Forman J, Damschroder L. Qualitative content analysis. In: Jacoby L, Siminoff LA, editors. Empirical methods for bioethics: a primer. Volume 11. Amsterdam: Elsevier; 2008. p. 39-62.

7. Klaus K. Content analysis: an introduction to its methodology. Thousand Oaks: Sage; 2004.

8. Kitson A, Harvey G, McCormack B. Enabling the implementation of evidence based practice: a conceptual framework. Quality in Health Care. 1998;7:149-58.

9. Stetler $C B$, Damschroder $L$, Helfrich CD, Hagedorn HJ. A guide for applying a revised version of the PARIHS framework for implementation. Implement Sci. 2011:6:99.

10. Colquhoun HL, Brehaut JC, Sales A, Ivers N, Grimshaw J, Michie S, et al. A systematic review of the use of theory in randomized controlled trials of audit and feedback. Implement Sci. 2013;8(66):1-8. doi:10.1186/1748-5908-8-66.

11. Davies P, Walker AE, Grimshaw JM. A systematic review of the use of theory in the design of guideline dissemination and implementation strategies and interpretation of the results of rigorous evaluations. Implement Sci. 2010;5(14):1-6. doi:10.1186/1748-5908-5-14.

12. Grol R, Bosch MC, Hulscher MEJ, Eccles MP, Wensing M. Planning and studying improvement in patient care: the use of theoretical perspectives. Milbank Q. 2007;85(1):93-138. doi:10.1111/j.1468-0009.2007.00478.x.

13. Tabak RG, Khoong EC, Chambers DA, Brownson RC. Bridging research and practice: models for dissemination and implementation research. Am J Prev Med. 2012:43(3):337-50. doi:10.1016/j.amepre.2012.05.024.

14. Grimshaw JM, Eccles MP, Lavis JN, Hill SJ, Squires JE. Knowledge translation of research findings. Implement Sci. 2012;7(50):1-17.

15. Novins DK, Green AE, Legha RK, Aarons GA. Dissemination and implementation of evidence-based practices for child and adolescent mental health: a systematic review. J Am Acad Child Adolesc Psychiatry. 2013;52(10):1009-1025.e18. doi:10.1016/j.jaac.2013.07.012.

16. Powell BJ, Proctor EK, Glass JE. A systematic review of strategies for implementing empirically supported mental health interventions. Res Soc Work Pract. 2014;24(2):192-212. doi:10.1177/1049731513505778.

17. Parmelli E, Flodgren G, Schaafsma ME, Baillie N, Beyer FR, Eccles MP. The effectiveness of strategies to change organizational culture to improve healthcare performance. Cochrane Database Syst Rev. 2011; 1. doi:10.1002/14651858.CD008315.pub2

18. Scheirer MA, Dearing JW. An agenda for research on the sustainability of public health programs. Am J Public Health. 2011;101(11):2059-67.

19. Stirman, SW, Kimberly J, Cook N, Calloway A, Castro F, Charns M. The sustainability of new programs and innovations: a review of the empirical literature and recommendations for future research. Implement Sci. 2012; $7(7): 17$.

20. Kitson AL. The need for systems change: reflections on knowledge translation and organizational change. JAN. 2009;65(1):217-28.
21. Aarons GA, Fettes DL, Sommerfeld DH, Palinkas LA. Mixed methods for implementation research: application to evidence-based practice implementation and staff turnover in community-based organizations providing child welfare services. Child Maltreat. 2012;17(1):67-79. doi:10.1177/1077559511426908.

22. Palinkas LA, Aarons GA, Horwitz S, Chamberlain P, Hurlburt M, Landsverk J. Mixed methods designs in implementation research. Adm Policy Ment Health. 2011;38:44-53. doi:10.1007/s10488-010-0314-z.

23. Raghavan R. The role of economic evaluation in dissemination and implementation research. In: Brownson RC, Colditz GA, Proctor EK, editors. Dissemination and implementation research in health: translating science to practice. New York: Oxford University Press; 2012. p. 94-113.

24. Vale L, Thomas R, MacLennan G, Grimshaw J. Systematic review of economic evaluations and cost analyses of guideline implementation strategies. Eur J Health Econ. 2007;8:111-21. doi:10.1007/s10198-007-0043-8.

25. Luke DA. Getting the big picture in community science: methods that capture context. Am J Community Psychol. 2005;35(3/4):185-200. doi:10.1007/s10464-005-3397-z.

doi:10.1186/s13012-015-0282-5

Cite this article as: Newman et al: Identifying priorities in knowledge translation from the perspective of trainees: results from an online survey. Implementation Science 2015 10.

\section{Submit your next manuscript to BioMed Central and take full advantage of:}

- Convenient online submission

- Thorough peer review

- No space constraints or color figure charges

- Immediate publication on acceptance

- Inclusion in PubMed, CAS, Scopus and Google Scholar

- Research which is freely available for redistribution 